$\because A-U R-95-9514 \overline{5}$

tiTle: AN APPLICATION OF MULTISURFACE PLASTICITY THEORY: YIELD SURFACES OF TEXTURED MATERIALS

AUTHOR(S): $\quad$ P(aul) J. Maudlin, T-3, Theoretical Division

S. I. Wright, CMS

U. F. Kocks, CMS

M(anjit) S. Sahota, T-3, Theoretical Division

SUBMITTED TO: Joint XV AIRAPT \& XXXIII EHPRG International Conference, Warsaw, Poland, September 11-15, 1995
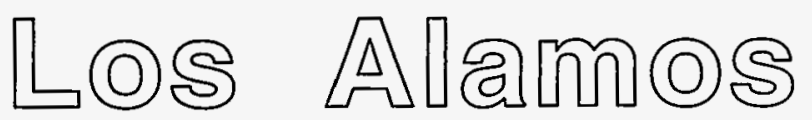

Los Alamos National Laboratory Los Alamos, New Mexico 87545

FORM NO. 836 R4 ST. NO. 2629 5/81 


\section{DISCLAIMER}

Portions of this document may be illegible in electronic image products. Images are produced from the best available original document. 


\title{
AN APPLICATION OF MULTISURFACE PLASTICITY THEORY: YIELD SURFACES OF TEXTURED MATERIALS
}

\author{
P. J. Maudlin, S. I. Wright, U. F. Kocks and M. S. Sahota \\ Los Alamos National Laboratory \\ Los Alamos, New Mexico 87545, USA
}

\begin{abstract}
Directionally dependent descriptions of the yield behavior of metals as determined by polycrystal plasticity computations are discrete in nature and, in principle, are available for use in large-scale application calculations employing multi-dimensional continuum mechanics codes. However, the practical side of using such detailed yield surfaces in application calculations contains some challenges in terms of algorithm development and computational efficiency. Discrete representations of yield as determined from Taylor-Bishop-Hill polycrystal calculations can be fitted or tessellated into a multi-dimensional piece-wise linear yield surface for subsequent use in constitutive algorithms for codes. Such an algorithm that utilizes an associated flow based multisurface plasticity theory has been implemented in the three dimensional EPIC code and is described in this effort.
\end{abstract}

\section{Introduction}

$X$-ray diffraction techniques can be used to measure the distribution of crystallographic orientations in a polycrystalline material(1). The resultant orientation distribution (OD) can then be then used to weight a set of discrete orientations to generate a representation of the material texture(2). This discrete representation of the measured texture can be probed in the context of a Taylor-Bishop-Hill polycrystal calculation with a set of incremental strain probes in order to form a set of deviatoric stress points that map out the material's yield surface(3). These stress points can be fitted or tessellated(4) into a multi-dimensional piecewise linear yield surface for subsequent use in a continuum code constitutive algorithm. Koiter(5) and later Simo(6) developed an associated flow based theory (multisurface plasticity) that can accept a piece-wise linear description of the yield envelope. A constitutive algorithm that utilizes this theory has been implemented in the three dimensional (3D) EPIC code and is described and illustrated below.

\section{Tessellation of Polycrystal Information}

In general, yield functions are five dimensional (5D) in terms of the deviatoric stress components $s_{i j}$, i.e.,

$$
\left[s_{i j}\right]=\left[\begin{array}{lll}
s_{11} & s_{12} & s_{13} \\
& s_{22} & s_{23} \\
& & s_{33}
\end{array}\right]
$$

where this tensor has five independent components (recall $s_{k k}=0$ ). Therefore the stress components $\left(s_{11}, s_{22}, s_{12}, s_{13}, s_{23}\right)$ define the general 5D space that needs to be spanned by some convex yield function, constraining the magnitude of the stress state during plastic flow. For the sample yield surface presented below, a 3D stress space $\left(s_{11}, s_{22}, s_{12}\right)$ is assumed, although the algorithm discussed in Sec. III is appropriate for the 5D problem.

Now consider the 3D case where a set of stress points are generated by repetitive polycrystal probes of a measured material OD. This set of points is tessellated (a linear fitting complete with associated connectivity) into a piece-wise surface in three space using a tessellation algorithm(4). An example of such a tessellation is shown in Fig. 1, which is a tantalum (BCC) yield surface corresponding to a rolling texture and thus closely approximates an orthotropic mechanical response. This surface is basically a linear interpolation of 647 stress points with $\mathrm{m}=1226$ linear functions or planes (in 5D say hyperplanes), the whole of which can be mathematically expressed (using indicial notation) as the set: 


$$
\left\{f^{\beta} \equiv \alpha_{i j}^{\beta} s_{i j}-\sigma^{\beta}=0, \beta=1,2, \ldots, m\right\}
$$

The linear functions appearing in Eq. (2) are expressed in normal form that defines the $\alpha_{i j}^{\beta}$ as coefficients of a vector normal to the hyperplane and $\sigma^{\beta}$ as the distance between the origin and the $\beta$ hyperplane.

Figure 1: Perspective view of a piece-wise linear representation of a tantalum yield surface constructed from polycrystal calculations that use experimental measurements of the material texture as the initial grain orientation basis. The $x, y, z$ axes shown are stress axes corresponding to $s_{11}, s_{22}, s_{12}$.

The yield function shown in Fig. 1 is actually only a normalized yield shape and thus needs to be scaled with some flow stress function $\sigma$ (in equivalent stress units) to obtain the absolute surface in deviatoric stress space, i.e.,

$$
s_{i j}=\tilde{s}_{i j} \sigma \frac{1}{\bar{M}}, \sigma^{\beta}=\tilde{\sigma}^{\beta} \sigma \frac{1}{\bar{M}}
$$

where the quantities over scored with a tilde represent normalized variables: the results of tessellated polycrystal calculations. The average Taylor Factor $\bar{M}$ appearing in Eqs. (3) and (4) corresponds in direction to that of the uniaxial stress data obtained to characterize the flow stress function $\sigma$; normalization of the polycrystal stress $\tilde{\mathrm{s}}_{\mathrm{ij}}$ is such that the uniaxial stress relationship between deviatoric stress and $\sigma$ can be recovered from Eq. (3).

\section{Multisurface Plasticity Algorithm}

If we now assume that the set of discontinuous piece-wise linear functions as represented by Eq. (2) is given, this yield surface can be utilized in an elastoplastic constitutive algorithm based on the multisurface plasticity theory of Koiter(5) and later Simo(6). This algorithm is modified here to facilitate its use in the framework of an explicit continuum code whose purpose is high-rate applications. The approach follows classical associated flow theory starting with a general anisotropic form of Hooke's law written in terms of a deviatoric stress rate and strain rate $\dot{e}_{i j}$ (deviatoric portion of the symmetrical part of the velocity gradient tensor):

$$
\dot{s}_{i j}=E_{i j k l} \dot{e}_{k l}^{e}
$$

where $E_{i j k l}$ is a symmetric elastic constant (stiffness) tensor. Assuming the standard practice of partitioning the strain-rate $\dot{e}_{i j}$ into elastic and plastic parts, we can rewrite Eq. (5) as

$$
\dot{s}_{i j}=E_{i j k l}\left(\dot{e}_{k l}-\dot{e}_{k l}^{p}\right)
$$

with a flow rule for the plastic part expressed as a summation of contributions from those linear functions which are active:

$$
\dot{e}_{i j}^{p}=\sum_{\beta=1}^{m_{a c t}} \dot{\lambda} \frac{\partial f^{\beta}}{\partial s_{i j}}
$$

Here $\dot{\lambda}^{\beta}$ is a time dependent proportionality scalar. Note that the stress gradients in Eq. (7) are just the constants $\alpha_{i j}^{\beta}$ since the individual $f^{\beta}\left(s_{i j}\right)$ functions are linear; thus we have for our particular choice of Eq. (2)

$$
\dot{e}_{i j}^{p}=\sum_{\beta=1}^{m_{a g e}} \dot{\lambda}^{\beta} \alpha_{i j}^{\beta}
$$

The next step is to enforce yield surface consistency by taking the time derivative of Eq. (2), assuming that the flow stress is constant over the explicit time step $\Delta t$ (this is good assumption as discussed in (7)), and substitute for the stress rate and the plastic strain rate via Eqs. (6) and (8): 


$$
\dot{f^{\beta}}=\frac{\partial f^{\beta}}{\partial s_{i j}} \dot{s}_{i j}=\alpha_{i j}^{\beta} E_{i j k l}\left(\dot{e}_{k l}-\sum_{\zeta=1}^{m_{a c}} \dot{\lambda} \dot{\zeta}_{i j}^{\zeta}\right)=0
$$

Now if the total strain rate $\dot{e}_{i j}$ is assumed to be a given (and constant over the time step), then yield surface consistency as represented by the right-hand portion of Eq. (9) is applied to each of the $m_{\text {act }}$ active hyperplanes, resulting in a system of $m_{a c t}$ equations to be solved for the $m_{a c t}$ unknowns $\dot{\lambda} \zeta$.

Most of the work associated with the use of this theory involves identifying the active hyperplanes out of a total population of hyperplanes that can be arbitrarily large. From the mathematical concept of linear independence, the number of active linear functions can't be any larger then the dimension of our stress solution domain, i.e., $m_{a c t} \leq \operatorname{dim}\left\{f^{\beta}\left(s_{i j}\right)\right\}$, which is $5 \mathrm{D}$ for the general case of Eq. (1) and 3D for the simpler case illustrated by Fig. 1. For the Fig. 1 case the stress state during plastic flow can reside on a vertex (intersection of three planes, thus three linear functions are active), on an edge (intersection of two planes, thus two linear functions are active) or anywhere on a single plane (one linear function active); the analogy for the general 5D case is also valid.

Therefore the algorithm proceeds by identifying the active linear functions with a final step to correct for numerical error, as discussed in more detail in (8).

\section{A Simple Rectangular Shear Test Problem}

A useful problem for checking the continuum code implementation of any constitutive algorithm is simple rectangular shear. A 1-cm-square quadrilateral planestrain element was modeled with the EPIC code using the Fig. 1 yield surface, a set of $E_{i j k l}$ for orthotropic rolled tantalum, and the multisurface plasticity algorithm presented above. Figure 2 shows stress history results from the EPIC simulation of the simple rectangular problem over $200 \%$ strain or $40 \mu \mathrm{s}$ of time $(t)$ for a shearing velocity of $1000 \mathrm{~m} / \mathrm{s}$. The non-smooth nature of this stress solution as the material flows plastically from a state of pure shear to one dominated by the normal components is a direct result of the discontinuous piece-wise nature of the yield surface; in contrast, if the surface were represented by single analytic function, then the stress solution would be smooth.

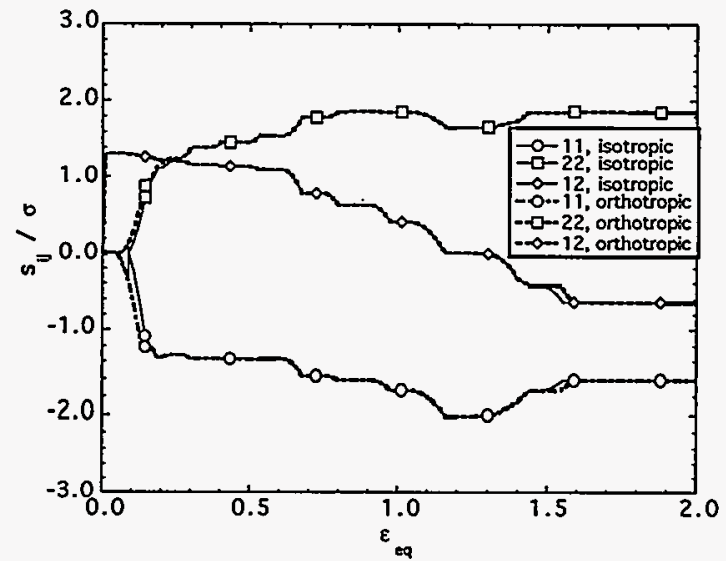

Figure 2: Material frame deviatoric stress components versus equivalent strain $\sqrt{2 \mathrm{e}_{\mathrm{ij}} \mathrm{e}_{\mathrm{ij}} / 3}$ for the simple rectangular shear problem where a constant value for the flow stress $\sigma$ has been assumed for convenience. Results using both isotropic and orthotropic elasticity are shown.

\section{References}

1. J. S. Kallend, U. F. Kocks, A. D. Rollett and H.-R. Wenk, Mat. Sci. and Engr., A132 1-11 (1991).

2. U. F. Kocks, J. S. Kallend and A. C. Biondo, Textures and Microstructures, 14-18 199-204 (1991).

3. G. R. Canova, U. F. Kocks and C. N. Tomé, J. Mech. Phys. Solids, 33 371-397 (1985).

4. M. S. Sahota, Proceed. Next Free-Lagrange Conf., Moran, Wyoming, 3-7 June, 1990.

5. W. T. Koiter, Quart. Appl. Math., 11, 350354 (1953).

6. J. C. Simo, J. G. Kennedy and S. Govindjee, Int. J. Num. Meth. Engr., 26, 2161-2185 (1988).

7. P. J. Maudlin and S. K. Schiferl,Comp. Meth. in Appl. Mech. and Engr. (accepted for publication, June, 1995).

8. P. J. Maudlin, S. I. Wright, U. F. Kocks and M. S. Sahota, Acta Metall, (submitted for publication, March 1995). 


\section{DISCLAIMER}

This report was prepared as an account of work sponsored by an agency of the United States Government. Neither the United States Government nor any agency thereof, nor any of their employees, makes any warranty, express or implied, or assumes any legal liability or responsibility for the accuracy, completeness, or usefulness of any information, apparatus, product, or process disclosed, or represents that its use would not infringe privately owned rights. Reference herein to any specific commercial product, process, or service by trade name, trademark, manufacturer, or otherwise does not necessarily constitute or imply its endorsement, recommendation, or favoring by the United States Government or any agency thereof. The views and opinions of authors expressed herein do not necessarily state or reflect those of the United States Government or any agency thereof.

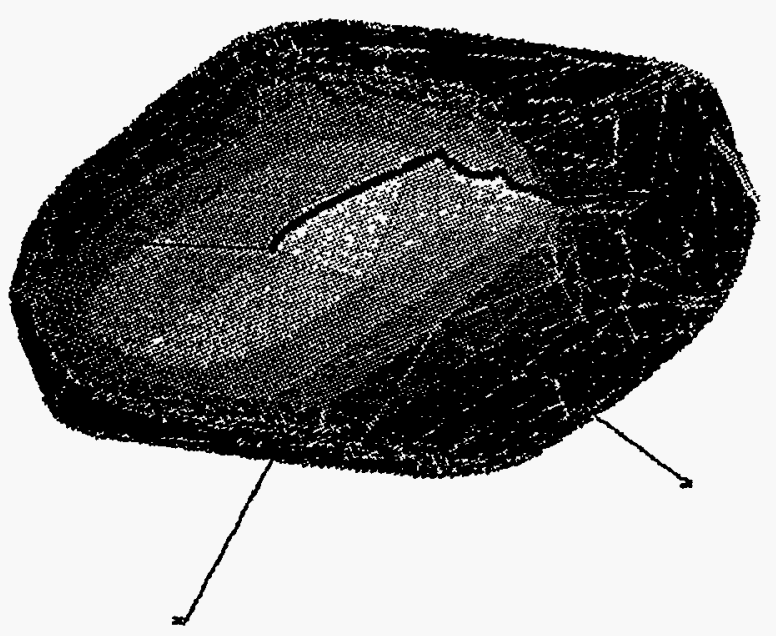

